(2) Open Access Full Text Article

REVIEW

\title{
Antibiotic optimization in the difficult-to-treat patient with complicated intra-abdominal or complicated skin and skin structure infections: focus on tigecycline
}

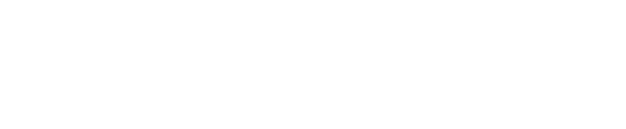

\section{Wanda C Reygaert}

Department of Biomedical Sciences, Oakland University William Beaumont School of Medicine, Rochester, MI, USA
Correspondence: Wanda C Reygaert Oakland University William Beaumont School of Medicine, Rochester, MI 48309, USA

Tel +I 2483702709

Fax + I 2483704060

Email reygaert@oakland.edu
Abstract: Complicated intra-abdominal and skin and skin structure infections are widely varied in presentation. These infections very often lead to an increase in length of hospital stay, with a resulting increase in costs and mortality. In addition, these infections may be caused by a wide variety of bacteria and are often polymicrobial with the possibility of the presence of antimicrobial-resistant strains, such as methicillin-resistant Staphylococcus aureus, vancomycinresistant enterococci, extended-spectrum $\beta$-lactamase strains (Escherichia coli, Klebsiella pneumoniae), and K. pneumoniae carbapenemase-producing strains. In combination with patients' immunosuppression or comorbidities, the treatment and management options for initial therapy success are few. Tigecycline, a new glycylcyline antimicrobial from the tetracycline drug class, represents a viable option for the successful treatment of these infections. It has been shown to have activity against a wide variety of bacteria, including the antimicrobial-resistant strains. As with all tetracycline drugs, it is not recommended for pregnant or nursing women. The potential side effects are those typical of tetracycline drugs: nausea, vomiting, and headaches. Drug-drug interactions are not expected, and renal function monitoring is not necessary.

Keywords: complicated intra-abdominal infections, complicated skin and skin structure infections, tigecycline

\section{Introduction}

Complicated intra-abdominal infections (cIAIs) and complicated skin and skin structure infections (cSSSIs) are responsible for increased morbidity and mortality in affected patients and lead to greatly increased health care costs due to extra treatment requirements and extended hospitalization. Failure to initiate a proper therapy regimen early in the course of treatment is also responsible for the increased morbidity and mortality. This could include failure to initiate antimicrobial therapy, administration of an inappropriate antimicrobial agent, or inappropriate dosing or monitoring of antimicrobial therapy. These issues most commonly arise when the infectious agent is unidentified or when there is a delay in identification. The initial therapy may well need to be a broadspectrum antimicrobial, and this drug (or drugs in combination) needs to have the highest efficacy possible. The ever increasing threat of antimicrobial resistance from all types of bacteria makes the choice of an initial antimicrobial agent a major challenge.

Tigecycline, a recently developed broad-spectrum antimicrobial agent, which is a derivative of minocycline, was designed to be able to overcome some of the major antimicrobial resistance issues and has been used clinically in Europe for 4 years and in 
the Uniteds States for 3 years. It is approved for use in both cIAIs and cSSSIs, and it shows promise for use in communityacquired (CA) pneumonia infections. Tigecycline has shown favorable antimicrobial activity against a wide range of bacteria including Gram-positive, Gram-negative, and anaerobic strains, some of these strains being those that exhibit serious antimicrobial resistance. ${ }^{1,2}$

In order to be able to properly manage cIAIs and cSSSIs, it is necessary to have an understanding of what these conditions encompass. An uncomplicated IAI usually involves only a single organ and the infection stays localized to that site. These infections are usually treated using only antibiotic therapy or, if necessary, surgical resection. In cIAIs, the infections spread beyond the local site, with a resulting peritonitis (or abscess). Peritonitis is further categorized as primary (no loss of integrity of the gastrointestinal [GI] tract), secondary (loss of GI tract integrity, usually by perforation or from infected viscera such as the appendix), and tertiary (recurrent infection following a primary or secondary peritonitis)..$^{3-6}$ In addition, secondary cIAIs are usually polymicrobial, with a potential mixture of Gram-negative and Gram-positive aerobic and anaerobic bacteria. There may be so many different or varied types of bacteria present, including some potentially antimicrobial-resistant strains that all may not be able to be isolated and identified. The most commonly isolated nonanaerobes are the Gram-negative Enterobacter species, E. coli, Klebsiella species, Proteus species, and Pseudomonas aeruginosa and the Gram-positive Enterococcus species, Staphylococcus species and Streptococcus species. The most commonly isolated anaerobes are Bacteroides species, Clostridium species, Fusobacterium species, and Peptostreptococcus species. Candida species may also be isolated..$^{7-11}$ Table 1 lists the common isolates from these infections.

SSSIs may be classified into four levels: (1) localized with no complications; (2) localized, but with comorbidity, or systemic and stable; (3) having toxic symptoms or no toxic symptoms with an unstable comorbidity; and (4) having sepsis syndrome or with other life-threatening infection. ${ }^{12}$ These infections are generally considered to be complicated if they fall into classes 2,3 , or $4 .{ }^{13}$ More generally, cSSSIs are described as those infections that have moved deeper into structures such as the fascia or muscles and/or require surgical intervention. ${ }^{14,15}$ Other pertinent divisions of cSSSIs may include CA vs hospital-acquired (HA) infections, chronic vs acute, localized vs diffuse, and non necrotizing vs necrotizing. ${ }^{15,16}$ Uncomplicated SSSIs are more likely to be monomicrobial, and the most common isolates from these
Table I Common isolates from intra-abdominal infections

\begin{tabular}{lll}
\hline Primary & Usually & Enterobacteria \\
peritonitis & monomicrobial & Staphylococci \\
& & Streptococci \\
Secondary & Usually & Nonanaerobes \\
peritonitis & polymicrobial & Enterobacter species \\
& & Enterococci \\
& Escherichia coli \\
& Klebsiella species \\
& Proteus species \\
& Pseudomonas aeruginosa \\
& Staphylococci \\
& Streptococci \\
& & Anaerobes \\
& Bacteroides species \\
& & Clostridium species \\
& & Fusobacterium species \\
& & Peptostreptococcus species \\
Tertiary & Candida species \\
peritonitis & Depends on cause & Enterococci \\
& & Pseudomonas aeruginosa \\
& & Staphylococci \\
& & Candida species \\
\hline
\end{tabular}

infections and from CA-cSSSIs are Gram-positive cocci (staphylococci, streptococci, enterococci). ${ }^{12,15,17,18}$ Complicated SSSIs and HA-cSSSIs are more likely to be polymicrobial (or monomicrobial if the isolate is an antimicrobial-resistant strain), ${ }^{19}$ with isolates including similar Gram-positive cocci plus varying isolates depending on the type and site of trauma involved. The most common additional isolates are members of the Enterobacteriaceae (E. coli, Enterobacter species, Klebsiella species, Proteus species, Serratia species) and P. aeruginosa. ${ }^{15,20,21}$ Table 2 lists the most common isolates from cSSSIs.

The types of organisms isolated and the severity of morbidity and mortality in cIAIs and cSSSIs are extremely varied and depend on additional risk factors. These factors fall into two general divisions: those associated with patient's status, such as comorbidities (eg, diabetes mellitus), critical illnesses of any kind, age (eg, infants, the elderly), immunocompetency (compromised: eg, human immunodeficiency virus [HIV] and transplant patients), liver and/or kidney disease, and vascular

Table 2 Most common bacterial isolates from cSSSIs

\begin{tabular}{ll}
\hline CA-cSSSIs & HA-cSSSIs \\
\hline Staphylococcus aureus & Staphylococcus aureus \\
Streptococcus pyogenes & Pseudomonas aeruginosa \\
Enterococcus species & Enterococcus species \\
& Escherichia coli \\
\hline
\end{tabular}

Abbreviations: cSSSIs, complicated skin and skin structure infections; CA-cSSSIs, community acquired-complicated skin and skin structure infections; HA-cSSSIs, hospital acquired-complicated skin and skin structure infections. 
sufficiency; and those associated with the type of causative trauma, and whether the trauma is CA or HA. ${ }^{21-24}$

\section{Impact on morbidity, mortality, and costs}

Studies performed on recently collected data from various health care facilities provide useful information on the seriousness of the issues involved in the treatment of cIAIs and cSSSIs. Because patients with cIAIs and cSSSIs are usually hospitalized, they are at high risk of developing an additional health care-associated infection (HAI). A study of data from 994 acute care hospitals across the United States in 2000 found that the hospitalized patients were at risk for what were termed "medical injuries" while in the hospital. These injuries included events such as accidental puncture or laceration, complications of anesthesia, iatrogenic pneumothorax, postoperative hemorrhage or hematoma, postoperative hip fracture, postoperative pulmonary embolism, postoperative sepsis, postoperative wound dehiscence, and transfusion reaction. Patients who developed postoperative sepsis averaged over 10 days each of excess hospitalization, resulting in an average of almost US\$58,000 each in extra health care costs and an average of almost $22 \%$ increase in mortality. Patients who experienced wound dehiscence averaged almost 10 extra days of hospitalization, over US\$40,000 in extra costs, and an increase in mortality rate of almost $10 \% .{ }^{25} \mathrm{~A}$ study on data from 283 hospitals in the United States in 2002 that analyzed HAIs categorized into five major types (surgical site, bloodstream, pneumonia, urinary tract infections, and other combined infections) found that an estimated 1.7 million HAIs resulted in nearly 99,000 deaths for that year. ${ }^{26}$ This number exceeded the number of deaths from any notifiable disease. ${ }^{27}$

Two other studies collected data on patients with IAI. The first study analyzed data from the Netherlands and found that hospitalized patients with complicated (secondary) IAIs who had not been given appropriate initial antibiotic treatment $(16 \%)$ had over a three fold higher risk of clinical failure than those given appropriate treatment. This resulted in an average increased hospital stay of over 7 days and an average excess in costs of over $€ 6,000 .^{28}$ The other study analyzed data from the year 2000 in the United States on patients who underwent intra-abdominal surgery and developed postoperative pneumonia. The data from 994 hospitals in 28 states showed that of the more than 600,000 patients that had intraabdominal surgery, over 13,000 developed pneumonia and almost $11 \%$ of them died. Developing pneumonia resulted in an average of over 11 days of extra hospitalization and over US \$31,000 in average excess costs, which was a mean increase in costs of $75 \%{ }^{29}$

A study on hospitalized patients with cSSSIs analyzed data from over 400 US hospitals during 2003-2004. The study included over 47,000 patients, almost 11,000 (nearly $23 \%$ ) of which experienced a failure of initial antibiotic therapy. This resulted in an average of over 5 days of extra hospitalization, an average of over US $\$ 5,000$ in excess costs, and a three fold increase in mortality. ${ }^{30}$

\section{Increasing problem of antimicrobial resistance}

An extremely important issue that adds to the complexity of management and treatment of cIAIs and cSSSIs is the ever-increasing problem of antimicrobial-resistant bacteria. Several of the most common isolates from these types of infections are bacteria that are at the forefront in the antimicrobial-resistance issue. These include methicillin-resistant S. aureus (MRSA), vancomycin-resistant enterococci (VRE), extended-spectrum $\beta$-lactamase (ESBL)-producing bacteria (E. coli and K pneumonia), K. pneumoniae carbapenemase (KPC)-producing bacteria (K. pneumonia, Enterobacter species, E. coli, and Pseudomonas species), P. aeruginosa (which has other antimicrobial capabilities), and anaerobic bacteria having various antimicrobial mechanisms (Bacteroides species and Clostridium species). Table 3 represents a list of antimicrobial agents that are not effective against these resistant organisms.

\section{Methicillin-resistant S. aureus}

The most common isolate from cSSSIs and a frequent isolate from cIAIs is $S$. aureus. This means that any antimicrobial mechanism in these bacteria could be a major management and treatment issue. The percentage of isolates of $S$. aureus that are methicillin-resistant strains has been increasing steadily since first discovered, with a current average in the United States of nearly $60 \% .{ }^{31}$ In addition, there is the issue of CA-MRSA strains vs HA-MRSA strains. Although the CA-MRSA usually are not resistant to other drug classes, the HA-MRSA have been found to be resistant to multiple drug classes, such as the aminoglycosides and fluoroquinolones, and a few isolates have recently been found to be resistant to vancomycin. ${ }^{32}$ The CA-MRSA, however, are most likely to produce the Panton-Valentine leukocidin, which is associated with cSSSIs that are highly virulent and with cases of necrotizing fasciitis. ${ }^{33} \mathrm{An}$ important variation in resistance among the CA-MRSA strains involves the main European 
Table 3 Antimicrobial agents that are not effective against resistant organisms

\begin{tabular}{|c|c|}
\hline \multicolumn{2}{|l|}{ MRSA } \\
\hline $\mathrm{CA}$ & $\beta$-lactam drugs \\
\hline \multirow[t]{8}{*}{$\mathrm{HA}$} & $\beta$-lactam drugs \\
\hline & Aminoglycosides \\
\hline & Fluoroquinolones \\
\hline & Oxazolidinones \\
\hline & Streptogamins \\
\hline & Tetracycline \\
\hline & Trimethoprim-sulfamethoxazole \\
\hline & Vancomycin - beginning \\
\hline \multirow[t]{11}{*}{ VRE } & Vancomycin \\
\hline & associated with ampicillin resistance \\
\hline & Aminoglycosides \\
\hline & Clindamycin \\
\hline & Erythromycin \\
\hline & Tetracycline \\
\hline & Trimethoprim-sulfamethoxazole \\
\hline & facilitate colonization \\
\hline & Aminoglycosides \\
\hline & Extended-spectrum cephalosporins \\
\hline & Fluoroquinolones \\
\hline \multirow[t]{4}{*}{ ESBLs } & Aminoglycosides \\
\hline & $\beta$-lactam drugs, including third-generation cephalosporins, \\
\hline & monobactams, and sometimes carbapenems (KPCs) \\
\hline & Sulfonamides \\
\hline \multirow[t]{6}{*}{ Anaerobes } & $\begin{array}{l}\beta \text {-lactam drugs - most increasing resistance to } \\
\text { cephalosporins }\end{array}$ \\
\hline & Chloramphenicol - some strains \\
\hline & Clindamycin - low to moderate resistance \\
\hline & Metronidazole - in the Gram-positives \\
\hline & Quinolones - low to moderate resistance \\
\hline & $\begin{array}{l}\text { Tetracycline - most } B \text {. fragilis strains, also found in } C \text {. } \\
\text { perfringens }\end{array}$ \\
\hline
\end{tabular}

Abbreviations: MRSA, methicillin-resistant S. aureus; CA, community-acquired; HA, hospital-acquired; VRE, vancomycin-resistant enterococci; ESBLs, extendedspectrum $\beta$-lactamases; KPCs, K. pneumoniae carbapenemases.

CA-MRSA strain, the ST80 strain. It has been isolated in several countries including Belgium, England, France, Germany, Switzerland, and the Netherlands. Isolates from areas that include Europe, Greece, and China have been shown to be highly resistant to tetracycline. The usual mechanism of resistance in these strains is via the $t e t K$ gene, which encodes an efflux pump. ${ }^{34-36}$ Although the tet $K$ gene confers tetracycline resistance, these isolates are usually susceptible to minocycline. ${ }^{37}$ These resistance issues severely limit the possibilities for antimicrobial therapy. Infections with MRSA also increase health care costs, length of hospital stay, and mortality in already complicated infections. ${ }^{38}$ It has been estimated that each year over 126,000 people are hospitalized with MRSA infections. These infections result in an average excess cost of US $\$ 20,000$ per case..$^{39}$ An extremely disturbing fact is that now more deaths per year in the United States are attributed to MRSA than those from tuberculosis and HIV/AIDS combined. ${ }^{40,41}$

\section{Vancomycin-resistant enterococci}

Enterococci are frequent isolates from cSSSIs and cIAIs. Approximately $95 \%$ of these isolates are Enterococcus faecalis and E. faecium strains. ${ }^{42}$ The majority of VRE strains are E. faecium, which are also highly resistant to ampicillin. VRE are believed to be natural colonizers in the GI tract, and infections are most often seen in patients who are immunocompromised and who have received antimicrobial therapy with multiple drugs. ${ }^{43,44}$ Enterococci have been found to be resistant to many types of antimicrobial agents, such as clindamycin, trimethoprim-sulfamethoxazole, aminoglycosides, and tetracyclines. In addition, it has been found that certain antimicrobials (extended-spectrum cephalosporins, fluoroquinolones) actually facilitate colonization of VRE, so the possibilities for antimicrobial therapy are extremely restricted. ${ }^{42-44}$ Clinical isolates of VRE are continuing to increase in numbers, with rates in the United States in the $20 \%-40 \%$ range. In Europe, the numbers are also increasing, but the overall rate is lower (around 10\%). It has been postulated that the higher resistance rates in the United States correlate with a higher use rate of vancomycin therapy. ${ }^{45,46}$ VRE infections also have a very serious impact on health care, with an average increase in hospital stay of over 6 days, an average increase in cost of almost US $\$ 13,000$ per infection, and an average increased mortality of $6 \% .{ }^{47}$

\section{Extended-spectrum $\beta$-lactamases}

Enzymes that work against the $\beta$-lactam drugs (penicillins, cephalosporins, monobactams, carbapenems) are known collectively as $\beta$-lactamases. The enzymes can be classified functionally into groups (the Bush-Jacoby-Medeiros classification scheme groups 1-4) and/or by the molecular makeup of their nucleotide and amino acid sequences (the Ambler groups A-D). ${ }^{48}$ Over 200 ESBLs, which occur in Gram-negative bacteria, are known, most of which are classified functionally into group 2 be and into Ambler group A. The enzymes in this group inactivate third-generation cephalosporins and monobactams but are usually inhibited by the $\beta$-lactamase inhibitor, clavulanic acid. ${ }^{49}$ The genes for ESBLs are usually located on plasmids, and these same plasmids often carry genes for aminoglycoside and sulfonamide resistance as well. ${ }^{50} \mathrm{ESBLs}$ are found most often in the Enterobacteriaceae, particularly in E. coli and K pneumoniae, and also in $P$. aeruginosa. ${ }^{49,51}$ These same organisms may also carry genes for producing other $\beta$-lactamases such 
as the AmpC-type enzymes and carbapenemases (such as the KPCs), which may not be inactivated by $\beta$-lactamase inhibitors. ${ }^{48,49,51}$ The ESBL-carrying organisms are among the most clinically important isolates from cIAIs and HA-cSSSIs. The most serious issue when dealing with these organisms is the consequence of inappropriate use of antimicrobial agents during initial therapy. Because the $\beta$-lactam drugs have activity against the structure of the peptidoglycan layer in the bacterial cell wall and Gram-negative bacteria (unlike Gram-positive bacteria) have a thin peptidoglycan layer that is somewhat protected by the much thicker outer lipopolysaccharide layer, it is probably best in complicated diseases to use antimicrobial agents from one or more of the other drug classes instead. The use of monotherapy or combination therapy with various $\beta$-lactam drugs has shown to result in treatment failure in many patients, with an accompanying increase in mortality. ${ }^{52-55}$ In addition, infections with ESBL organisms increase the length of hospital stay with a resulting increase in costs..$^{52,56}$

\section{Anaerobes}

Anaerobic bacteria are sometimes significant isolates from cIAIs. Due to the production of $\beta$-lactamases, most anaerobic bacteria are highly resistant to the penicillin drugs, with low to moderate, but increasing resistance to the cephalosporins and emerging resistance to the carbapenems. Most anaerobes are also resistant to the aminoglycosides. Clindamycin resistance is low to moderate and increasing, especially among Bacteroides fragilis strains. ${ }^{57-61} \mathrm{~B}$. fragilis, which is the most common anaerobic clinical isolate, is also highly resistant to tetracycline (up to $90 \%$ of strains), has shown moderate resistance to quinolones and fluoroquinolones, and varied low-level resistance to chloramphenicol and metronidazole. Clostridium species have shown a high level of resistance to clindamycin (67\%), and some tetracycline isolates have been isolated. ${ }^{57,59}$

\section{Management issues in patients with comorbidities}

There are a number of variables that come into play during the management of complicated infections that affect patient's outcome. These potential additional factors can be directly responsible for increased length of hospital stay, increased costs, and increased mortality. Some of these factors are influenced by the variables in the complicated infections themselves, and some factors, such as preexisting comorbidities, necessitate changes in management and treatment. In addition to the general factor of the site of any initiating trauma, cSSSIs are also influenced by major factors such as an initial infection with MRSA or whether the major trauma was burn related; and cIAIs are influenced by additional major factors such as organ repair, removal, and/or transplant. Whenever immune system depression is involved, whether as a result of an infection or condition, or because immunosuppressing drugs are part of the therapy, the risk of complicated infection and mortality is much higher. It is interesting to note that patients with cIAIs that occur as a result of complicated appendicitis have a lower mortality rate than those with cIAIs from other causes, and patients who acquire an IAI in the hospital (nosocomial IAIs) have a higher mortality rate than those acquired in the community. ${ }^{62}$

If the initial infection in a cSSSI is caused by MRSA, then the management issues depend on whether the MRSA strain is a CA-MRSA or an HA-MRSA. CA-MRSA tend to be resistant only to $\beta$-lactam drugs, whereas HA-MRSA often are resistant to multiple drug classes. In addition, the CA-MRSA strains usually carry an additional virulence factor, the Panton-Valentine leukocidin, which can lead to a necrotizing infection. Management of these MRSA infections requires appropriate initial antimicrobial therapy, which in turn requires expedited identification and susceptibility data. Failure to implement the proper antimicrobial therapy early in these infections can lead to a significant increase in mortality. MRSA infections are also a major cause of cSSSIs and cIAIs when the initial trauma was from a skin injury or from surgery.

There are an estimated two million serious burn injuries per year in the United States. ${ }^{63}$ If the patient is a burn victim, especially with third-degree burns, he or she is at high risk for development of infection in the burn wounds. Because severely burned individuals are usually admitted to a health care facility immediately, they are at high risk for nosocomial infections. The complications in burn wounds that are major issues include the amount of loss of skin area and whether there is also inhalation damage. ${ }^{64,65}$ Severe damage to the epithelial layers of the skin triggers an immune response that leads to immune system dysfunction, which results in a much higher risk for infection. ${ }^{63,66}$ These complications and infections can result in the spread of infection into the blood stream and can lead to organ system failure. ${ }^{63-65}$ The most common isolate from burn infections is $P$. aeruginosa. Following in close second place as an isolate is Acinetobacter baumannii. Both of these organisms are usually found to be resistant to multiple classes of antimicrobial agents. ${ }^{65,67}$ These complications can result is severely limited treatment options for patients with burn injuries. 


\section{Patients with comorbidities}

Patients with special comorbidities, such as HIV infection or cancer, have special treatment issues. Because of a potential crippling of the immune system, HIV patients are at high risk for developing many types of opportunistic infections with fungi, parasites, viruses, and bacteria. The most common bacterial infections are caused by Mycobacterium species. HIV patients are given prophylactic treatment for opportunistic infections based on their response to antiretroviral treatment. An increase in $\mathrm{CD}^{+}$lymphocyte number to certain specified levels may indicate that prophylactic treatment is no longer needed. Studies have shown that when HIV patients have acceptable lymphocyte levels, they are at low risk for developing opportunistic infections. ${ }^{68-70}$ If an HIV patient develops a cSSSI or cIAI, then the treatment will depend on his or her immune status and the particular bacteria involved. HIV patients are also at high risk of developing certain types of cancers. Another therapy issue occurs with the antiretroviral drug didanosine. Because it interferes with the absorption of fluoroquinolones and tetracyclines, the patient needs to be instructed to stagger the two drug types with 2 hours between the alternate doses. ${ }^{71,72}$

The presence of cancer as a comorbidity can have a multitude of effects on patients with cSSSIs and cIAIs. As with HIV, patients with cancer that require chemotherapy are at risk for development of some of the same types of opportunistic infections. The most common opportunistic bacterial isolates are Gram-positive streptococci and staphylococci. ${ }^{73,74}$ Prophylactic treatment is not usually recommended, and antimicrobial choices are based not only on the causative organism but also on the level of neutropenia and the presence of a fever or other symptoms of an infection. ${ }^{74,75}$ Because cancer can be a localized or systemic disease, the treatment and management of patients with cSSSIs and cIAIs will need to be tailored for the needs of the individual patient. There is a lack of information on antimicrobial drug interactions in cancer patients. However, the Infectious Diseases Society of America recommends in its guidelines for antimicrobial therapy in cancer patients that to avoid the possibility of renal toxicity, cisplatin, amphotericin B, cyclosporine, vancomycin, and the aminoglycosides should not be used in combination. ${ }^{75}$

There are many general concerns in transplant patients because they are put on immunosuppressive therapy, so they are at high risk of developing infections. They have to be monitored carefully for any symptoms. Each type of infection has to be dealt with on an individual basis. There are some therapy concerns when using cyclosporine (and related drugs). Cyclosporine has an additive nephrotoxicity when used with aminoglycosides, it can lower the drug metabolism of erythromycin, and can increase the drug metabolism of rifampin. $^{72}$

\section{Patients with diabetes}

Patients with diabetes have a high risk of developing infections in their lower extremities. Excess glucose present in the blood stream can result in impaired microvascular circulation and peripheral motor neuropathy, which may lead to an increased infection risk. Any skin-damaging injury or surgery is a huge infection risk factor. In addition, because of the circulation and neuropathy issues, infected tissues do not heal as easily as in nondiabetic patients. An estimated 25\% of adult diabetics (the risk increases with age) will develop a foot infection, and this infection will lead to amputations and increased mortality. ${ }^{76-78}$ The usual bacterial isolate from mild to moderate foot infections are Gram-positive cocci, with an accompanying risk of MRSA infection. More severe infections may be polymicrobial and also may include members of the Enterobacteriaceae and anaerobes. ${ }^{76,79}$ Even without any additional health issues such as surgery, diabetic patients have a good chance of having to undergo antimicrobial therapy. There can be some issues related to antimicrobial drugs and the medications that the diabetic patient takes to lower his or her glucose levels competing with each other in metabolic pathways. This can lead to hypoglycemia or hyperglycemia, so a diabetic patient should be monitored carefully while on antimicrobial therapy. ${ }^{80-82}$

\section{Patients with renal or hepatic failure}

Patients with impaired renal function are not only at risk for development of an infection but also the loss of renal function can also directly affect the efficiency of any antimicrobial therapy. The extent of the effect depends on the amount of lost function and the type of antimicrobial agent used. Antimicrobial compounds that are hydrophilic are generally excreted through the kidney. Those that are lipophilic may be metabolized first (often by the liver) and then eliminated ${ }^{83,84}$ In addition, patients who undergo some type of dialysis will likely lose some of any antimicrobial drug during that process. These patients need to be carefully monitored, if possible, for drug levels. ${ }^{85-87}$ Patients who do not maintain optimal drug levels will then have increased length of hospital stay and a higher risk of mortality. ${ }^{88}$ Patients who have to undergo kidney transplantation are also at high risk of infection because of the necessary immunosuppression therapy. 
Impaired liver function can lead to a multitude of problems. Patients with acute liver failure (ALF) are at high risk of infection, and this will commonly lead to multiple system organ failure, which is the most common cause of death in these patients. ${ }^{89}$ Approximately $50 \%$ of ALF patients will undergo liver transplantation. Immunosuppressive therapy after transplantation puts them at high risk of infection, and bacterial and fungal infections are responsible for nearly one-third of deaths that occur after transplantation. For this reason, patients who are to undergo a liver transplant are usually given extensive prophylactic therapy. ${ }^{90}$ Cirrhosis of the liver, which is most often caused by hepatitis $\mathrm{C}$ infection or alcoholic liver disease, is responsible for up to $80 \%$ of adult liver transplants. ${ }^{91}$ Because the liver is responsible for metabolizing many clinically important drugs (including lipophilic antimicrobial agents), when there is decreased function, the metabolism of these drugs can be greatly reduced, and patients have to be carefully monitored because of this. ${ }^{83,92}$

\section{Antibiotic selection}

There are certain considerations that need to be addressed before determining any initial, or in the case of previously failed therapy, additional antimicrobial therapy for any type of infection. The first consideration for deciding on antimicrobial therapy should be CA or HA. Even with the same organism (eg, MRSA), the susceptibilities can vary greatly between these two parameters. Other factors to take into consideration include chronic or acute infection, patient's history of similar infections, patient's history of antimicrobial use (including any known antimicrobial allergies), and treatment history for the current infection. With these facts in hand, the physician can be on the watch for potential antimicrobial-resistant organisms. Then, the patient's health status needs to be considered. Is the patient immunosuppressed, does he or she have diabetes or other comorbidities, or is he or she on chemotherapy? Then, with a careful examination of the patient and proper sampling of the infection site, a Gram stain report (and later a culture report, if ordered) can provide the physician with important information about the infection: is there only one organism present (predominately) or is it a polymicrobial infection; are bacteria only present and/or fungi; Gram-positive and/or Gram-negative? In addition, the physician has to assess the infection for signs that suggest an anaerobic bacterial infection. Now, the physician can make an informed decision on the choice of antimicrobial therapy, which may necessitate initial use of a broad-spectrum drug (or drug combination if the infection is polymicrobial), then changing to a more specific drug when information on organisms' identification and drug susceptibility is available.

\section{Complicated skin and skin structure infections}

Although most cSSSIs are caused by Gram-positive cocci, there is a difference in the organisms isolated from CA-cSSSIs and HA-cSSSIs, such as MRSA. In addition, some of the Gram-negative bacteria are common isolates from HA-cSSSIs. It is best to use this parameter as a guide for initial antimicrobial therapy options, especially if a Gram stain report is not available or is delayed. Infection with Streptococcus species usually respond favorable to standard semisynthetic penicillin or early generation cephalosporins. However, if the patient is allergic to penicillin and because the group A streptococci are showing resistance to erythromycin, a lincosamide such as clindamycin could be used. Because of increasing resistance to these drugs, it might be best to use drugs from antimicrobial classes that have different foci (eg, cell-wall inhibition, protein synthesis inhibition) in combination. ${ }^{19,93}$

The other causative organisms mentioned in Table 2 are among the serious antimicrobial resistance organisms, and therefore have to be treated with extreme care. When deciding on initial antimicrobial therapy, before definitive organism antimicrobial susceptibility data are available, it might be prudent to err on the side of caution. Basing the initial therapy on the assumption that the causative organism might be a resistant one may be the safer option. When susceptibility data become available, the antimicrobial therapy can then be tailored for the specific organism. If the physician waits to treat resistant organisms until they have been identified, the infection may then be out of control and mortality rates will increase greatly.

Because the incidence of MRSA among S. aureus isolates is so high (nearly two of every three isolates in the United States), it may be best to treat all isolates as if they were MRSA. Antimicrobial therapy can be based on CA (not usually multidrug resistant) vs HA (often multidrug resistant) guidelines. Most CA-MRSA strains are susceptible to tetracyclines, fluoroquinolones, trimethoprim or sulfamethoxazole, clindamycin, and vancomycin. Vancomycin is probably not needed for CA-MRSA infections and perhaps should not be used as initial therapy. Clindamycin therapy has issues because resistance can be induced in some MRSA strains. For serious HA-MRSA infections, vancomycin is the current accepted standard treatment. ${ }^{19,93,94}$ 
Serious infections with VRE are a major treatment issue. The percentage of Enterococcus species isolates that are vancomycin resistant is increasing at an alarming rate. Recent antimicrobial susceptibility studies recommend the use of the newer glycopeptide or glycylcyline drugs. ${ }^{95,96}$

Gram-negative bacteria isolated from HA-cSSSIs may be strains that are ESBLs or possibly P. aeruginosa, which is naturally resistant to multiple drugs. The potential ESBL organisms, E. coli and K. pneumoniae, may also be resistant to multiple drugs. The suggested antimicrobial therapy for ESBL organisms is to use carbapenem, fluoroquinolone, or aminoglycoside drugs, alone or in combination. ${ }^{48,49}$ In addition, some K. pneumoniae isolates may produce carbapenemases (KPCs), so carbapenem drugs would not be an option for such isolates. Potential antimicrobials that can be used for $P$. aeruginosa infection include piperacillin or ticarcillin, some third-generation and fourth-generation cephalosporins, aztreonam, active carbapenems, ciprofloxacin or levofloxacin, and certain aminoglycosides (gentamicin, tobramycin, amikacin). ${ }^{23,97}$

Anaerobes may sometimes be isolated from serious diabetic foot infections. The most commonly isolated anaerobe is Clostridium perfringens, which is a rapid grower and a potential cause of necrotizing and "gas gangrene" infections. Because it grows so rapidly, it is important to initiate antimicrobial therapy quickly. Recommended therapy includes penicillin $\mathrm{G}$ and clindamycin in combination. Patients who are allergic to penicillin are usually given clindamycin or metronidazole. ${ }^{98}$

\section{Complicated intra-abdominal infections}

Many of the same organisms that cause cSSSIs are also isolated from cIAIs with similar antimicrobial therapy recommendations. Gram-positive cocci (Streptococcus species, MRSA, VRE) may have the same antimicrobial resistance issues. Some of the most common Gram-negative isolates are potential ESBL strains or $P$. aeruginosa, with similar antimicrobial therapy recommendations. Potential anaerobic isolates include Clostridium species, with similar recommended therapy, and Bacteroides species, which may have resistance to multiple drugs. The current recommended antimicrobial therapy for Bacteroides species is metronidazole or chloramphenicol..$^{59,60}$ In addition to all the potential antimicrobial-resistant strains, cIAIs are most often polymicrobial infections, not only with Gram-positive and Gram-negative but also with nonanaerobes, anaerobes, and potentially with yeast such as Candida species or other fungi. This means that some sort of complicated combination therapy will probably be needed. The risk of improper initial antimicrobial therapy failing is very high in these infections and leads to greatly increased mortality rates. ${ }^{9}$

\section{Clinical utility of tigecycline}

Tigecycline is currently approved for intravenous use in cSSSIs and cIAIs. It is a glycylcyline drug semisynthetically derived from the tetracycline drug, minocycline, by addition of a 9-t-butylglycylamido group. It binds reversibly to the $30 \mathrm{~S}$ bacterial ribosomal subunit and blocks the tRNAs from being able to enter the ribosome A site, effectively inhibiting protein synthesis. ${ }^{99-102}$ The glycylamido side chain allows tigecycline to overcome the usual resistance mechanisms against the tetracycline drugs (which are produced by acquisition of the various tet resistance genes), resulting in a stronger binding affinity than that of minocycline (three fold) and tetracycline (20-fold), allowing it to bind well to TetMmodified ribosomes, and keeping tigecycline from being transported out of the bacteria via a tetracycline-specific efflux pump. ${ }^{99,103,104}$ Given intravenously in a usual dosing regimen of $100 \mathrm{mg}$ initially and then $50 \mathrm{mg}$ every 12 hours, tigecycline exhibits a large volume of distribution $(7-9 \mathrm{~L} / \mathrm{kg})$ with good tissue penetration. Concentrations of tigecycline in various tissues compared with serum concentrations are typically 38 -fold in the gallbladder, 8-9-fold in the lung, and over two fold in the colon, with a steady state plasma concentration of $0.87 \mu \mathrm{g} / \mathrm{mL}$ and a mean terminal half-life of 37-38 hours. ${ }^{105}$ It has a rapid uptake into polymorphonuclear neutrophils, which peaks at approximately 1 hour after dosing and is 20-30-fold higher than the extracellular concentration. ${ }^{105,106}$ Tigecycline is not metabolized in the liver and is independent of the CYP450 system, so it does not affect drugs that are metabolized by these enzymes, which means that there is no required monitoring of renal function, and there is a low potential for drug-drug interactions. ${ }^{99,105,107,108}$ It is excreted predominately unchanged, with about $59 \%$ excreted in feces and $22 \%-32 \%$ excreted in urine. ${ }^{104}$ The only adverse effects were those similar to the tetracycline drugs: nausea, vomiting, and headache. ${ }^{99,107}$ Costs must be taken into consideration when choosing antimicrobial therapy, but when faced with potentially life-threatening infections, cheapest is not always best. The typical daily cost for tigecycline therapy is more than piperacillin or tazobactam but less than linezolid, daptomycin, or meropenem. ${ }^{108}$

Susceptibility tests have shown tigecycline to have a very broad spectrum of activity against bacteria, including the most serious threats for drug resistance in cSSSIs and cIAIs. These include Gram-positive cocci - MRSA, VRE; 
Gram-negativecocci- ESBLs, KPCs; and anaerobes. These data suggest that tigecycline could be used as monotherapy, or in drug combinations, for initial antimicrobial therapy, with an excellent chance that this therapy would be successful. This could lead to greatly improved infection management, lower costs, and decreased mortality. There were a few bacteria that exhibited a reduced susceptibility, and tigecycline monotherapy is probably not recommended for them. Those organisms include Proteus mirabilis, the indole-positive Proteeae (Proteus vulgaris, Morganella morganii, Providentia species), and $P$. aeruginosa when isolated from the lungs of cystic fibrosis patients. ${ }^{100,101,109}$ It is also not recommended to use for pregnant or nursing women as the tetracycline drugs can cross the placenta and may be present in high concentrations in breast milk. ${ }^{99}$ Table 4 lists the bacteria that are susceptible to tigecycline.

Table 4 Organisms against which tigecycline demonstrated favorable bacteriostatic activity

\begin{tabular}{l}
\hline Gram-positive \\
Corynebacterium jeikeium \\
Enterococcus species - including VRE \\
Listeria monocytogenes \\
Staphylococcus species - including MRSA \\
Streptococcus species \\
Gram-negative \\
Enterobacteriaceae - including ESBLs, KPCs, and other $\beta$-lactamases \\
Citrobacter species \\
Enterobacter species \\
Escherichia coli \\
Klebsiella species \\
Salmonella species \\
Serratia marcescens \\
Shigella species \\
Non-Enterobacteriaceae \\
Acinetobacter species \\
Haemophilus influenzae \\
Moraxella species \\
Neisseria species \\
Stenotrophomonas maltophilia \\
Anaerobes \\
Bacteroides species \\
Clostridium difficile \\
Clostridium perfringens \\
Fusobacterium species \\
Peptostreptococcus species \\
Porphyromonas species \\
Prevotella species \\
Atypionibacterium acnes \\
Chlamydophila pneumoniae \\
Mycoplasma species \\
\hline
\end{tabular}

Abbreviations: VRE, vancomycin-resistant enterococci; MRSA, methicillinresistant S. aureus; ESBLs, extended-spectrum $\beta$-lactamases; KPCs, K pneumoniae carbapenemases.

\section{Conclusion}

cIAIs and cSSSIs can be very challenging to manage and treat. These infections vary greatly in severity based not only on general patients' health (immunosuppression, comorbidities, etc) but also on the wide variety of bacteria that can be involved (Gram-positive, Gram-negative, anaerobes). These infections are often polymicrobial and may contain any of the most feared antimicrobial-resistant strains of bacteria (MRSA, VRE, ESBLs, $\mathrm{KPCs}$ ). Identifying a proper antimicrobial treatment regimen for initial therapy is vital because failure of initial treatment correlates with greatly increased costs and higher mortality rates.

The new glycylcyline antimicrobial, tigecycline, is a broadspectrum antibiotic and has shown activity against a wide variety of bacteria (including antimicrobial-resistant strains). Tigecycline shows great promise for use in cSSSIs and cIAIs especially when a broad-spectrum drug is needed for initial antimicrobial therapy. Even though it is only approved for intravenous use, this results in greater tissue penetration, which is highly important in these types of infections. Side effects may present, but are not more serious than any of the tetracycline drugs. The daily cost for this drug is moderately high, but that cost can easily be offset when initial treatment success translates to shorter hospital stays and decreased mortality. Tigecycline seems likely to be able to fill a gap in antimicrobial therapy that has been widening, and perhaps will help ease the anxiety of physicians who face the treatment and management of patients with antimicrobial-resistant infections.

\section{Disclosure}

The author reports no conflicts of interest in this work.

\section{References}

1. Townsend ML, Pound MW, Drew RH. Tigecycline in the treatment of complicated intra-abdominal and complicated skin and skin structure infections. Ther Clin Risk Manag. 2007;3(6):1059-1070.

2. Nicolau DP. Management of complicated infections in the era of antimicrobial resistance: the role of tigecycline. Expert Opin Pharmacother. 2009; 10(7):1213-1222.

3. McClean KL, Sheehan GJ, Harding GK. Intraabdominal infection: a review. Clin Infect Dis. 1994;19(1):100-116.

4. Menichetti F, Sganga G. Definition and classification of intra-abdominal infections. J Chemother. 2009;21 Suppl 1:3-4.

5. Solomkin JS, Mazuski JE, Bradley JS, et al. Diagnosis and management of complicated intra-abdominal infection in adults and children: guidelines by the Surgical Infection Society and the Infectious Diseases Society of America. Surg Infect (Larchmt). 2010;11(1):79-109.

6. Sartelli M. A focus on intra-abdominal infections. World J Emerg Surg 2010;5:9.

7. Barie PS. Management of complicated intra-abdominal infections. J Chemother. 1999;11(6):464-477.

8. Krobot K, Yin D, Zhang Q, et al. Effect of inappropriate initial empiric antibiotic therapy on outcome of patients with community-acquired intraabdominal infections requiring surgery. Eur J Clin Microbiol Infect Dis. 2004;23(9):682-687. 
9. Blot S, de Waele JJ. Critical issues in the clinical management of complicated intra-abdominal infections. Drugs. 2005;65(12): $1611-1620$.

10. Napolitano LM, Sawyer RG, Stevens DL. Contemporary approaches to complex infections. Supplement to Contemporary Surgery. 2006 Dec;1-16. Available from: http://www.contemporarysurgery.com/ uploadedFiles/CSSupp_Infections_1206.pdf. Accessed May 3, 2010.

11. Brook I. Microbiology and management of abdominal infections. Dig Dis Sci. 2008;53(10):2585-2591.

12. Eron LJ, Lipsky BA, Low DE, et al. Managing skin and soft tissue infections: expert panel recommendations on key decision points. J Antimicrob Chemother. 2003;52 Suppl 1:i3-i17.

13. Eisenstein BI. Treatment challenges in the management of complicated skin and soft-tissue infections. Clin Microbiol Infect. 2008;14 Suppl 2: $17-25$.

14. Nichols RL. Optimal treatment of complicated skin and skin structure infections. J Antimicrob Chemother. 1999;44 Suppl A:19-23.

15. Fung HB, Chang JY, Kuczynski S. A practical guide to the treatment of complicated skin and soft tissue infections. Drugs. 2003;63(14): $1459-1480$

16. DiNubile MJ, Lipsky BA. Complicated infections of skin and skin structures: when the infection is more than skin deep. $J$ Antimicrob Chemother. 2004;53 Suppl 2:ii37-ii50.

17. Love BL. Management of complicated skin and soft tissue infections in hospitalized patients. US Pharm. 2007;32(4):HS5-HS12.

18. Lipsky BA, Weigelt JA, Gupta V, et al. Skin, soft tissue, bone, and joint infections in hospitalized patients: epidemiology and microbiological, clinical, and economic outcomes. Infect Control Hosp Epidemiol. 2007;28(11):1290-1298.

19. May AK, Stafford RE, Bulger EM, et al. Treatment of complicated skin and soft tissue infections. Surg Infect (Larchmt). 2009; 10(5):467-499.

20. Moet GJ, Jones RN, Biedenbach DJ, et al. Contemporary causes of skin and soft tissue infections in North America, Latin America, and Europe: report from the SENTRY Antimicrobial Surveillance Program (1998-2004). Diagn Microbiol Infect Dis. 2007;57(1):7-13.

21. Raghavan M, Linden PK. Newer treatment options for skin and soft tissue infections. Drugs. 2004;64(15):1621-1642.

22. Podnos YD, Jimenez JC, Wilson SE. Intra-abdominal sepsis in elderly persons. Clin Infect Dis. 2002;35(1):62-68.

23. Ki V, Rotstein C. Bacterial skin and soft tissue infections in adults: a review of their epidemiology, pathogenesis, diagnosis, treatment and site of care. Can J Infect Dis Med Microbiol. 2008;19(2): 173-184.

24. Breen JO. Skin and soft tissue infections in immunocompetent patients. Am Fam Physician. 2010;81(7):893-899.

25. Zhan C, Miller MR. Excess length of stay, charges, and mortality attributable to medical injuries during hospitalization. JAMA. 2003;290(14):1868-1874

26. Klevens RM, Edwards JR, Richards CL Jr, et al. Estimating health care-associated infections and deaths in US hospitals, 2002. Public Health Rep. 2007;122(2):160-166.

27. Groseclose SL, Brathwaite WS, Hall PA; Centers for Disease Control and Prevention. Summary of notifiable diseases - United States, 2002. MMWR Morb Mortal Wkly Rep. 2004;51(53):1-84.

28. Sturkenboom MC, Goettsch WG, Picelli G, et al. Inappropriate initial treatment of secondary intra-abdominal infections leads to increased risk of clinical failure and costs. $\mathrm{Br} J$ Clin Pharmacol. 2005;60(4):438-443.

29. Thompson DA, Makary MA, Dorman T, Pronovost PJ. Clinical and economic outcomes of hospital acquired pneumonia in intra-abdominal surgery patients. Ann Surg. 2006;243(4):547-552.

30. Edelsberg J, Berger A, Weber DJ, et al. Clinical and economic consequences of failure of initial antibiotic therapy for hospitalized patients with complicated skin and skin-structure infections. Infect Control Hosp Epidemiol. 2008;29(2):160-169.

31. Reygaert W. Methicillin-resistant Staphylococcus aureus (MRSA): prevalence and epidemiology issues. Clin Lab Sci. 2009;22(2):111-114.
32. Woodford N, Livermore DM. Infections caused by gram-positive bacteria: a review of the global challenge. J Infect. 2009;59 Suppl 1:S4-S16.

33. Reygaert W. Methicillin-resistant Staphylococcus aureus (MRSA): molecular aspects of antimicrobial resistance and virulence. Clin Lab Sci. 2009;22(2):115-119.

34. Tristan A, Bes M, Meugnier H, et al. Global distribution of PantonValentine leukocidin-positive methicillin-resistant Staphylococcus aureus, 2006. Emerg Infect Dis. 2007;13(4):594-600.

35. Vourli S, Vagiakou H, Ganteris G, et al. High rates of communityacquired, Panton-Valentine leukocidin (PVL)-positive methicillinresistant S. aureus (MRSA) infections in adult outpatients in Greece. Euro Surveill. 2009;14(2):p11=19089

36. Liu Y, Kong F, Zhang X, et al. Antimicrobial susceptibility of Staphylococcus aureus isolated from children with impetigo in China from 2003 to 2007 shows community-associated methicillin-resistant Staphylococcus aureus to be uncommon and heterogeneous. Br J Dermatol. 2009;161(6):1347-1350.

37. Trzcinski K, Cooper BS, Hryniewicz W, Dowson CG. Expression of resistance to tetracyclines in strains of methicillin-resistant Staphylococcus aureus. J Antimicrob Chemother. 2000;45(6):763-770.

38. Klevens RM, Morrison MA, Nadle J, et al. Invasive methicillinresistant Staphylococcus aureus infections in the United States. JAMA. 2007;298(15):1763-1771

39. Institute for Healthcare Improvement. Protecting 5 Million Lives From Harm. 2006 Dec. Available from: http://www.ihi.org/IHI/Programs/ Campaign/. Accessed Jul 31, 2008.

40. Klevens RM, Edwards JR, Tenover FC, et al. Changes in the epidemiology of methicillin-resistant Staphylococcus aureus in intensive care units in US hospitals, 1992-2003. Clin Infect Dis. 2006;42(3):389-391.

41. Boucher HW, Corey GR. Epidemiology of methicillin-resistant Staphylococcus aureus. Clin Infect Dis. 2008;46 Suppl 5:S344-S349.

42. Rice LB. Emergence of vancomycin-resistant enterococci. Emerg Infect Dis. 2001;7(2):183-187.

43. Rice LB, Hutton-Thomas R, Lakticova V, et al. Beta-lactam antibiotics and gastrointestinal colonization with vancomycin-resistant enterococci. J Infect Dis. 2004;189(6):1113-1118.

44. Rice LB. Antibiotics and gastrointestinal colonization by vancomycinresistant enterococci. Eur J Clin Microbiol Infect Dis. 2005;24(12): 804-814.

45. Klare I, Konstabel C, Mueller-Bertling S, et al. Spread of ampicillin/ vancomycin-resistant Enterococcus faecium of the epidemic-virulent clonal complex-17 carrying the genes esp and hyl in German hospitals. Eur J Clin Microbiol Infect Dis. 2005;24(12):815-825.

46. Abele-Horn M, Vogel U, Klare I, et al. Molecular epidemiology of hospital-acquired vancomycin-resistant enterococci. J Clin Microbiol. 2006;44(11):4009-4013.

47. Carmeli Y, Eliopoulos G, Mozaffari E, Samore M. Health and economic outcomes of vancomycin-resistant enterococci. Arch Intern Med. 2002;162(19):2223-2228

48. Nicasio AM, Kuti JL, Nicolau DP. The current state of multidrugresistant gram-negative bacilli in North America. Ann Pharmacother. 2007;41(6):1077-1081.

49. Rupp ME, Fey PD. Extended spectrum beta-lactamase (ESBL)producing Enterobacteriaceae: considerations for diagnosis, prevention and drug treatment. Drugs. 2003;63(4):353-365.

50. Paterson DL. Resistance in gram-negative bacteria: enterobacteriaceae. Am J Infect Control. 2006;34(5 Suppl 1):S20-S28.

51. Bradford PA. Extended-spectrum beta-lactamases in the 21st century: characterization, epidemiology, and detection of this important resistance threat. Clin Microbiol Rev. 2001;14(4):933-951.

52. Lautenbach E, Patel JB, Bilker WB, et al. Extended-spectrum betalactamase-producing Escherichia coli and Klebsiella pneumoniae: risk factors for infection and impact of resistance on outcomes. Clin Infect Dis. 2001;32(8):1162-1171.

53. Du B, Long Y, Liu H, et al. Extended-spectrum beta-lactamaseproducing Escherichia coli and Klebsiella pneumoniae bloodstream infection: risk factors and clinical outcome. Intensive Care Med. 2002; 28(12):1718-1723. 
54. Anderson DJ, Engemann JJ, Harrell LJ, et al. Predictors of mortality in patients with bloodstream infection due to ceftazidime-resistant Klebsiella pneumoniae. Antimicrob Agents Chemother. 2006; 50(5):1715-1720.

55. Tumbarello M, Sanguinetti M, Montuori E, et al. Predictors of mortality in patients with bloodstream infections caused by extended-spectrumbeta-lactamase-producing Enterobacteriaceae: importance of inadequate initial antimicrobial treatment. Antimicrob Agents Chemother. 2007;51(6):1987-1994.

56. Schwaber MJ, Navon-Venezia S, Kaye KS, et al. Clinical and economic impact of bacteremia with extended-spectrum-beta-lactamaseproducing Enterobacteriaceae. Antimicrob Agents Chemother. 2006;50(4):1257-1262.

57. Rasmussen BA, Bush K, Tally FP. Antimicrobial resistance in anaerobes. Clin Infect Dis. 1997;24 Suppl 1:S110-S120.

58. Labbé AC, Bourgault AM, Vincelette J, et al. Trends in antimicrobial resistance among clinical isolates of the Bacteroides fragilis group from 1992 to 1997 in Montreal, Canada. Antimicrob Agents Chemother. 1999;43(10):2517-2519.

59. Hecht DW. Prevalence of antibiotic resistance in anaerobic bacteria: worrisome developments. Clin Infect Dis. 2004;39(1):92-97.

60. Liu CY, Huang YT, Liao CH, et al. Increasing trends in antimicrobial resistance among clinically important anaerobes and Bacteroides fragilis isolates causing nosocomial infections: emerging resistance to carbapenems. Antimicrob Agents Chemother. 2008;52(9):3161-3168.

61. Snydman DR, Jacobus NV, McDermott LA, et al. Lessons learned from the anaerobe survey: historical perspective and review of the most recent data (2005-2007). Clin Infect Dis. 2010;50 Suppl 1:S26-S33.

62. Inui T, Haridas M, Claridge JA, Malangoni MA. Mortality for intraabdominal infection is associated with intrinsic risk factors rather than the source of infection. Surgery. 2009;146(4):654-661.

63. Schwacha MG. Macrophages and post-burn immune dysfunction. Burns. 2003;29(1):1-14

64. Dries DJ. Management of burn injuries - recent developments in resuscitation, infection control and outcomes research. Scand J Trauma Resusc Emerg Med. 2009;17(1):14.

65. Branski LK, Al-Mousawi A, Rivero H, et al. Emerging infections in burns. Surg Infect (Larchmt). 2009;10(5):389-397.

66. Avni T, Levcovich A, Ad-El DD, et al. Prophylactic antibiotics for burns patients: systematic review and meta-analysis. BMJ. 2010;340:c241.

67. Keen EF III, Robinson BJ, Hospenthal DR, et al. Incidence and bacteriology of burn infections at a military burn center. Burns. 2010;36(4):461-468.

68. Gallant JE, Moore RD, Chaisson RE. Prophylaxis for opportunistic infections in patients with HIV infection. Ann Intern Med. 1994;120(11):932-944.

69. Kaplan JE, Hanson D, Dworkin MS, et al. Epidemiology of human immunodeficiency virus-associated opportunistic infections in the United States in the era of highly active antiretroviral therapy. Clin Infect Dis. 2000;30 Suppl 1:S5-S14.

70. Masur H, Kaplan JE, Holmes KK; US Public Health Service; Infectious Diseases Society of America. Guidelines for preventing opportunistic infections among HIV-infected persons - 2002. Recommendations of the U.S. Public Health Service and the Infectious Diseases Society of America. Ann Intern Med. 2002;137(5 Pt 2):435-478.

71. Burman WJ, Jones BE. Treatment of HIV-related tuberculosis in the era of effective antiretroviral therapy. Am J Respir Crit Care Med. 2001;164(1):7-12.

72. Katzung B, Masters S, Trevor A. Basic and Clinical Pharmacology. 11th ed. Chicago: McGraw-Hill; 2009.

73. Zinner SH. Changing epidemiology of infections in patients with neutropenia and cancer: emphasis on gram-positive and resistant bacteria. Clin Infect Dis. 1999;29(3):490-494.

74. Sullivan KM, Dykewicz CA, Longworth DL, et al. Preventing opportunistic infections after hematopoietic stem cell transplantation: the Centers for Disease Control and Prevention, Infectious Diseases Society of America, and American Society for Blood and Marrow Transplantation Practice Guidelines and Beyond. Hematology. 2001:392-421.
75. Hughes WT, Armstrong D, Bodey GP, et al. 2002 guidelines for the use of antimicrobial agents in neutropenic patients with cancer. Clin Infect Dis. 2002;34(6):730-751.

76. Cunha BA. Antibiotic selection for diabetic foot infections: a review. J Foot Ankle Surg. 2000;39(4):253-257.

77. Khanolkar MP, Bain SC, Stephens JW. The diabetic foot. QJM. 2008;101(9):685-695.

78. Nelson SB. Management of diabetic foot infections in an era of increasing microbial resistance. Curr Infect Dis Rep. 2009;11(5):375-382.

79. Rao N, Lipsky BA. Optimising antimicrobial therapy in diabetic foot infections. Drugs. 2007;67(2):195-214.

80. Griffin JP, D’Arcy PF. A Manual of Adverse Drug Interactions. 5th ed. The Netherlands: Elsevier; 1997.

81. Kaufman MB, Simionatto C. A review of protease inhibitor-induced hyperglycemia. Pharmacotherapy. 1999;19(1):114-117.

82. Owens RC Jr, Ambrose PG. Antimicrobial safety: focus on fluoroquinolones. Clin Infect Dis. 2005;41 Suppl 2:S144-S157.

83. Pea F, Viale P, Pavan F, Furlanut M. Pharmacokinetic considerations for antimicrobial therapy in patients receiving renal replacement therapy. Clin Pharmacokinet. 2007;46(12):997-1038.

84. Vilay AM, Churchwell MD, Mueller BA. Clinical review: drug metabolism and nonrenal clearance in acute kidney injury. Crit Care. 2008;12(6):235.

85. Bouman CS. Dosing of antimicrobial agents in critically-ill patients with acute kidney injury and continuous venvenous haemofiltration. Acta Clin Belg Suppl. 2007;(2):365-370.

86. Kuang D, Verbine A, Ronco C. Pharmacokinetics and antimicrobial dosing adjustment in critically ill patients during continuous renal replacement therapy. Clin Nephrol. 2007;67(5):267-284.

87. Mushatt DM, Mihm LB, Dreisbach AW, Simon EE. Antibiotic dosing in slow extended daily dialysis. Clin Infect Dis. 2009;49(3):433-437.

88. Reynvoet E, Vandijck DM, Blot SI, et al. Epidemiology of infection in critically ill patients with acute renal failure. Crit Care Med. 2009;37(7):2203-2209.

89. Stravitz RT. Critical management decisions in patients with acute liver failure. Chest. 2008;134(5):1092-1102.

90. O'Grady JG. Postoperative issues and outcome for acute liver failure. Liver Transpl. 2008;14 Suppl 2:S97-S101.

91. Moon DB, Lee SG. Liver Transplantation. Gut Liver. 2009; 3(3):145-165.

92. Jacob A, Zhou M, Wu R, Wang P. The role of hepatic cytochrome P-450 in sepsis. Int J Clin Exp Med. 2009;2(3):203-211.

93. Stevens DL, Bisno AL, Chambers HF, et al. Practice guidelines for the diagnosis and management of skin and soft-tissue infections. Clin Infect Dis. 2005;41(10):1373-1406.

94. Vinh DC, Embil JM. Severe skin and soft tissue infections and associated critical illness. Curr Infect Dis Rep. 2006;8(5):375-383.

95. Hasper D, Schefold JC, Baumgart DC. Management of severe abdominal infections. Recent Pat Antiinfect Drug Discov. 2009;4(1):57-65.

96. Eliopoulos GM. Microbiology of drugs for treating multiply drug-resistant Gram-positive bacteria. J Infect. 2009;59 Suppl 1:S17-S24.

97. Pappas G, Saplaoura K, Falagas ME. Current treatment of pseudomonal infections in the elderly. Drugs Aging. 2009;26(5):363-379.

98. Vinh DC, Embil JM. Severe skin and soft tissue infections and associated critical illness. Curr Infect Dis Rep. 2007;9(5):415-421.

99. Zhanel GG, Homenuik K, Nichol K, et al. The glycylcyclines: a comparative review with the tetracyclines. Drugs. 2004;64(1):63-88.

100. Rossi F, Andreazzi D. Overview of tigecycline and its role in the era of antibiotic resistance. Braz J Infect Dis. 2006;10(3):203-216.

101. Doan TL, Fung HB, Mehta D, Riska PF. Tigecycline: a glycylcycline antimicrobial agent. Clin Ther. 2006;28(8):1079-1106.

102. Hawkey P, Finch R. Tigecycline: in-vitro performance as a predictor of clinical efficacy. Clin Microbiol Infect. 2007;13(4):354-362.

103. Olson MW, Ruzin A, Feyfant E, et al. Functional, biophysical, and structural bases for antibacterial activity of tigecycline. Antimicrob Agents Chemother. 2006;50(6):2156-2166.

104. Peterson LR. A review of tigecycline - the first glycylcycline. Int $J$ Antimicrob Agents. 2008;32 Suppl 4:S215-S222. 
105. da Silva LM, Nunes Salgado HR. Tigecycline: a review of properties, applications, and analytical methods. Ther Drug Monit. 2010;32(3):282-288.

106. MacGowan AP. Tigecycline pharmacokinetic/pharmacodynamic update. J Antimicrob Chemother. 2008;62 Suppl 1:i11-i16.

107. Rose WE, Rybak MJ. Tigecycline: first of a new class of antimicrobial agents. Pharmacotherapy. 2006;26(8):1099-1110.
108. Stein GE, Craig WA. Tigecycline: a critical analysis. Clin Infect Dis. 2006;43(4):518-524.

109. Fritsche TR, Sader HS, Stilwell MG, et al. Potency and spectrum of tigecycline tested against an international collection of bacterial pathogens associated with skin and soft tissue infections (2000-2004). Diagn Microbiol Infect Dis. 2005;52(3):195-201.

\section{Publish your work in this journal}

Therapeutics and Clinical Risk Management is an international, peerreviewed journal of clinical therapeutics and risk management, focusing on concise rapid reporting of clinical studies in all therapeutic areas, outcomes, safety, and programs for the effective, safe, and sustained use of medicines. This journal is indexed on PubMed Central, CAS,
EMBase, Scopus and the Elsevier Bibliographic databases. The manuscript management system is completely online and includes a very quick and fair peer-review system, which is all easy to use. Visit http://www.dovepress.com/testimonials.php to read real quotes from published authors.

Submit your manuscript here: http://www.dovepress.com/therapeutics-and-clinical-risk-management-journal 